\title{
Antimicrobial Treatment of Mycobacterium ulcerans Infection
}

\author{
Till Frederik Omansen, Tjip S. van der Werf, \\ and Richard Odame Phillips
}

\section{$1 \quad$ Historical Aspects}

MacCallum and coworkers described Buruli ulcer (BU) as an infectious disease caused by Mycobacterium ulcerans in Victoria, Australia. They first considered the skin lesions in their patients to be caused by tuberculosis or leprosy, when they observed numerous acid-fast bacilli in the biopsy specimens [1]. The typical duration of illness was between 1 and 2 years; treatment was essentially surgical. With the advent of chemotherapy for tuberculosis [2-4], and later for leprosy, doctors made individual attempts to treat the lesions with anti-tuberculosis and anti-leprosy drugs. The anecdotal evidence suggested poor or no response to chemotherapy with rifampicin monotherapy [5], despite the fact that in vitro susceptibility of 33 strains of M. ulcerans was as good as for M. tuberculosis [6]. A randomized clinical trial by the British Medical Research Council in Buruli county (now called Nakasongola; Uganda) failed to show any benefit from clofazimine, a drug then first marketed for leprosy [7]. A small-sized trial with cotrimoxazole (18 participants; 12 evaluable) was inconclusive [8]. A small-sized randomized study in Côte d'Ivoire compared a

\footnotetext{
T. F. Omansen

Department of Tropical Medicine, Bernhard Nocht Institute for Tropical Medicine, Hamburg, Germany

I. Department of Medicine, University Medical Center Hamburg-Eppendorf, Hamburg, Germany

T. S. van der Werf $(\bowtie)$

Department of Internal Medicine, Division of Infectious Diseases, and Pulmonary Diseases \& Tuberculosis, University of Groningen, University Medical Centre Groningen,

Groningen, The Netherlands

e-mail: t.s.van.der.werf@umcg.nl

R. O. Phillips

Department of Medicine, Kwame Nkrumah University of Science \& Technology, Komfo

Anokye Teaching Hospital, Kumasi Center for Collaborative Research, Kumasi, Ghana
} 
combination of dapsone and rifampicin with placebo; the follow-up was limited; the ulcer size decreased slightly faster in the intervention group but the baseline characteristics of both groups differed, and the study did not allow to draw any firm conclusions about the effectiveness of these drugs [9]. By the turn of the millennium, the discrepancy between in vitro efficacy of rifampicin [6] or clarithromycin [10] and lack of clinical response prompted to stressing the need for well-designed and well-powered drug trials, but in the meantime, to also improve early detection and surgical treatment [11].

One important question that comes to mind when looking back at earlier studies that failed to show effectiveness of antimicrobial treatment for BU is how the clinical response was assessed. Even long before the chemical structure of the toxin mycolactone was discovered [12], it was realized that a toxin secreted by M. ulcerans was responsible for the extensive tissue necrosis [13, 14] as well as for the immune suppression observed and replicated in experimental animals, using a culture filtrate of $M$. ulcerans [15]. Radical surgical excision was to some extent effective but at the cost of tissue loss, and the need for plastic surgery, e.g., split-skin or full-thickness skin grafting. Reported recurrence rates following surgical treatment were variable; one follow-up study from Benin reported $6.1 \%$ recurrence in a subset of patients $(66 / 150 ; 44 \%)$ followed up to 7 years after surgery [16]. In a case series of 346 patients operated in three centers in Côte d'Ivoire, the recurrence rate was $17.1 \%$ [17]. A large difference in recurrence rates was reported from two centers in Ghana; $21 / 45(47 \%)$ of patients had a recurrence in one hospital, as compared to $6 / 33(18 \%)$ in the other hospital [18]. In a case series from the Bas Congo, DR Congo, of 51 patients seen over 2 consecutive years, 14 (39\%) had recurrent disease after previous surgery [19]. Lesions at a pre-ulcerative stage can be excised successfully with primary closure, however a 1 -year recurrence rate of $16 \%$ was observed in a cohort of 50 patients in Ghana [20].

With increased understanding of the dominant role of the secreted toxin mycolactone in the pathogenesis of Buruli ulcer, and realizing that perhaps the best measure of efficacy of antimicrobial treatment might be complete healing without recurrence, the effectiveness of antimicrobial treatment might have been underestimated in the past. Indeed with killing of M. ulcerans in lesions, as exemplified by the beneficial effects of different classes of antimicrobials in vitro [6,10,21], mycolactone secretion might stop, but the impact on tissue damage including necrosis would conceivably take more time than in any other infectious disease. Likewise, there was increased understanding of the profound effects of mycolactone on the immune system, with little local [22] and systemic inflammation [15]. Yet another factor perhaps blurring the observation of response to antimicrobial therapy is a common paradoxical increase in lesion size, development of new lesions, and increased inflammation following antimicrobial therapy [23-25]. Apart from the in vitro studies $[6,10,21]$, several studies in animal models had shown the efficacy of anti-mycobacterial drug combinations [26-28].

A formal proof-of-concept study to evaluate the potential of antimicrobial treatment without additional surgery was the landmark study conducted in Ghana, 
under the umbrella of the WHO Global Buruli Ulcer Initiative [29]. Patients having non-ulcerated lesions suspected to be M. ulcerans infection were randomly allocated to $0,4,8$ or 12 weeks of antimicrobial treatment with a combination of streptomycin and rifampicin; later a 5 th group receiving 2 weeks of treatment was added; the total number of study participants was 21 . Lesions were subsequently surgically removed and submitted for culture, PCR and histopathology. As no bacteriological confirmation tests were carried out prior to the start of therapy, some of the lesions remained bacteriologically unconfirmed to be Buruli ulcer disease; none of the patients receiving at least 4 weeks of treatment had viable bacilli by culture of their excised lesion; none had recurrent disease 12 months after start of treatment. Although some questions remained unanswered, this study provided the first robust evidence that antimicrobial treatment alone was able to sterilize non-ulcerated lesions of M. ulcerans infection, with no recurrence or positive culture in patients treated for at least 4 weeks with the antimicrobial combination [29].

With the introduction of antimicrobial treatment, a more conservative, less aggressive approach of surgery in addition to antimicrobial treatment was subsequently advocated [30-33].

For over 10 years now, the WHO has recommended antimicrobial treatment as the primary treatment modality. Below, we systematically discuss the literature on antimicrobial susceptibility of $M$. ulcerans in vitro and in animal models; and the accumulated evidence for effectiveness as well as safety (i.e., adverse reactions) of antimicrobial treatment regimens emerging from clinical studies.

\section{Antimicrobial Susceptibility of M. ulcerans}

For the review of in vitro activity of antimicrobial agents against $M$. ulcerans, we used a systematic approach searching the literature in PubMed. Using '(Buruli OR ulcerans) AND (antibiotic OR antimicrobial OR in-vitro OR susceptible)', we retrieved 520 unique results that we further analyzed for duplicates and consistent reporting. We cross-searched references obtained from articles we analyzed.

The most widely used antimicrobials for M. ulcerans infection have been rifampin, streptomycin and clarithromycin. M. ulcerans is a phylogenetically close relative of $M$. tuberculosis and $M$. leprae and has evolved from a common $M$. marinum-like ancestor [34]. Subsequently, many attempts to treat $M$. ulcerans infection have been undertaken seeking to use and repurpose antibiotics active against $M$. tuberculosis and $M$. leprae. To estimate efficacy in vitro, the usual way to screen these agents is by determining the minimal inhibitory concentration (MIC) of inoculates of $M$. ulcerans in mycobacterial culture media. Next, inoculation of viable $M$. ulcerans in the mouse footpad is a well-established in vivo model to assess efficacy. The final test is eventually to test these agents in patients affected by Buruli ulcer disease. Such studies are extremely challenging, as the majority of potential study 
participants are patients (many being children) that live in underprivileged circumstances with limited access to health care, and generally low educational background [35-37].

\subsection{Ansamycins/Rifamycins}

Ansamycins or rifamycins act on mycobacteria by inhibiting RNA synthesis through interfering with bacterial RNA-polymerase. Rifampin is the rifamycin most widely used in the treatment of Buruli ulcer. The drug has a strong bactericidal effect and an MIC of approximately $0.5 \mu \mathrm{g} / \mathrm{ml}$ [38-40]. Rifampin is administered orally at a dose of $10 \mathrm{mg} / \mathrm{kg}$ to humans to treat Buruli ulcer and is the backbone-drug of most regimens evaluated to date. Other rifamycins that have been shown to kill M. ulcerans are the rifampin-analogues rifabutin [26] and rifapentine [41]. Rifapentine is highly active against $M$. ulcerans with an MIC of $0.125-0.5 \mu \mathrm{g} / \mathrm{ml}[41,42]$. Because of its longer half-life time compared to rifampin, an intermitted rifapentine-based regimen with only two or three times weekly administration of antibiotics has been tested in mice [41]. Such an intermittent regimen is suggested to allow outpatient management and to simplify clinical management. However intermittent regimens also bear the risk of confusion and low compliance and patients being subsequently lost during treatment. Even though antibiotic resistance is not a major concern in the slowly replicating organism $M$. ulcerans, rifampicin resistance has been detected in rare instances [43, 44]. In-vivo, resistant mutants were isolated after monotherapy with rifampin in mice, which should be avoided in the clinical setting. The resistance was conferred by mutations in the rpoB gene of M. ulcerans [45]. Albeit uncommon and of unknown clinical significance, researchers in Ghana found 5.7$11.4 \%$ of isolates from their study districts to be resistant to $40 \mu \mathrm{g} / \mathrm{ml}$ rifampin [40]. Yet another rifamycin tested in M. ulcerans is rifabutin. Rifabutin has an MIC of $0.1-0.4 \mu \mathrm{g} / \mathrm{ml}$ against $M$. ulcerans [39].

\subsection{Aminoglycosides: Streptomycin}

Streptomycin is an injectable aminoglycoside with a strong bactericidal effect on $M$. ulcerans and one of the first agents ever shown to be effective against this organism $[46,47]$. It is a protein synthesis inhibitor that hinders binding of formyl-methionyltRNA to the bacterial ribosomal 30S subunit. In the mouse model, streptomycin remains one of the most active agents against $M$. ulcerans and is used as intramuscular injection of $15 \mathrm{mg} / \mathrm{kg}$ in humans [48]. In single drug in vitro and in vivo studies, streptomycin was more active than amikacin and linezolid, but less active than rifampicin and moxifloxacin [49]. In preclinical studies, streptomycin-containing regimens outperform all other commonly used drug combinations both in terms of colony-forming units remaining in infected mouse footpads and recurrence rate. However, the need to inject streptomycin is very impracticable in the rural African setting where Buruli ulcer is most common. It bears the risk of parenteral 
(needle-associated) infection and also threatens patient compliance due to fear of painful, daily injections, especially in children. Besides, streptomycin causes ototoxicity as well as nephrotoxicity as observed in patients followed after having been enrolled in the BURULICO trial [50].

\subsection{Amikacin}

Amikacin is another aminoglycoside that has been suggested for use in the treatment of patients with Buruli ulcer. It is highly active against M. ulcerans with an MIC of $0.5-1 \mu \mathrm{g} / \mathrm{ml}$ [21] and was shown to be equally efficient in the mouse model [26]. In a study testing a series of isolates, the MIC ranged between $0.25-1$ (mean, $0.65) \mu \mathrm{g} / \mathrm{ml}$ [49]. Amikacin was as effective as streptomycin in the M. ulcerans mouse model [26].

\subsection{Macrolides: Clarithromycin}

Clarithromycin is a protein synthesis inhibitor of the macrolide family. It reversibly binds to the $23 \mathrm{~S}$ rRNA on the 50 s ribosomal subunit and subsequently prevents polypeptide synthesis. It acts mainly as a bacteriostatic drug in M. ulcerans therapy. The MIC of clarithromycin against M. ulcerans is approximately $0.12 \mu \mathrm{g} / \mathrm{ml}[10$, $26,51,52]$. Clarithromycin is well tolerated, it can be taken orally and it is widely available. It has been shown to be non-inferior when replacing streptomycin after 4 weeks in the 8 week rifampin-streptomycin regimen while treating small lesions [36]. Following preliminary results of a randomized controlled trial (ClinicalTrials. gov identifier: NCT01659437) comparing 8 weeks rifampicin plus streptomycin with 8 weeks rifampicin plus clarithromycin, the latter regimen is now recommended by WHO. ${ }^{1}$

\subsection{Azithromycin}

Azithromycin is a macrolide antimicrobial agent active against a variety of organisms. The mean MIC against a collection of different strains was $0.39 \mu \mathrm{g} / \mathrm{ml}$ [40]. However when $100 \mathrm{mg}$ of azithromycin was administered to mice infected with $M$. ulcerans, only a modest bacteriostatic activity was observed [53].

\subsection{Fluoroquinolones}

Various fluoroquinolones such as ciprofloxacin, ofloxacin and moxifloxacin have been shown to be active against $M$. ulcerans with MICs ranging from 0.25 to $1 \mu \mathrm{g} / \mathrm{ml}$

\footnotetext{
${ }^{1}$ http://www.who.int/neglected_diseases/events/WHO_BU_TAG_2017_report.pdf?ua=1.
} 
[21]. Fluoroquinolones are bacterial DNA gyrase inhibitors. They impede DNA replication and transcription by hindering the DNA gyrase-catalyzed super-coiling of double-stranded DNA. In Australia, Buruli ulcer patients are frequently treated with rifampicin in combination with a fluoroquinolone, such as ciprofloxacin [54]. Such fluoroquinolones are administered orally and have favorable pharmacokinetic properties such as good tissue penetration. However, there is some concern about the use of fluoroquinolones in children; in Australia most Buruli ulcer patients are adults, most of them elderly, whereas in Africa where most of the cases are reported, the median age of patients is around 15 years. Fluoroquinolones may cause serious arthropathy in children and minors. However the evidence about increased toxicity in the young is incomplete. Although caution remains warranted, fluoroquinolones have increasingly been used in children without significantly increased adverse events or toxicity $[55,56]$. The most potent fluoroquinolone against $M$. ulcerans is sparfloxacin, with an MIC at $0.25 \mu \mathrm{g} / \mathrm{ml}$ in most strains tested [21]. The MIC of ofloxacin in tested strains of $M$. ulcerans was $1.26 \mu \mathrm{g} / \mathrm{ml}$ [40]; in another study, ofloxacin was least active of all fluoroquinolones tested with an MIC of $2 \mu \mathrm{g} / \mathrm{ml}$ [21]. Moxifloxacin (MIC $0.14 \mu \mathrm{g} / \mathrm{ml}$ ), sitafloxacin (MIC 0.125$0.5 \mu \mathrm{g} / \mathrm{ml}$ ) and prulifloxacin (MIC $1-3 \mu \mathrm{M}$ ) are also effective $[49,57,58]$. Of note, fluoroquinolones have increasingly been used in Ghana, one of the countries highly burdened with Buruli ulcer; an alarming rate of fluoroquinolone-resistant E. coli was noticed [59].

\subsection{Clofazimine}

Clofazimine is an anti-leprosy drug that has emerged as a critically important sterilizing drug in the treatment of multi-drug resistant tuberculosis. Its effect on mycobacteria is delayed but strongly bactericidal [60]. The mechanism of action is multifactorial, complex and not fully understood. As a prodrug, it is reduced by type $2 \mathrm{NADH}$-quinone oxidoreductase resulting in the release of bactericidal quantities of reactive oxygen species. It is then believed to competitively inhibit menaquinone, a crucial electron acceptor in the mycobacterial respiration chain [61]. Clofazimine possesses also some poorly understood anti-inflammatory activity for which it is used in conditions like severe acne or discoid lupus erythematosus [62]. These anti-inflammatory properties might also be part of its mode of action in mycobacterial infections where tissue damage by inflammatory processes is a hallmark. The MIC for various strains of M. ulcerans was $2.19 \mu \mathrm{g} / \mathrm{ml}$ on average [40]. As mentioned above, in an early trial clofazimine monotherapy was inefficacious in human Buruli ulcer patients [7]. In mice, clofazimine in combination with rifampicin was very effective with relapse-free cure following a 6-weeks oral regimen [63]. Clofazimine causes yellow-orange skin-discoloration as well as gastrointestinal disturbances as adverse effects [64]. Newer clofazimine analogs with reduced accumulation and thus less risk for skin discoloration are under development [58]. 


\subsection{Dapsone}

Dapsone is an anti-leprosy drug that has widely been used. Activity against $M$. ulcerans is moderate - the MIC ranged between 2.0 and $4.0 \mu \mathrm{g} / \mathrm{ml}$ in one study [39] and the mean MIC was $0.94 \mu \mathrm{g} / \mathrm{ml}$ in another study [40].

\subsection{Doxycycline}

Doxycycline, a tetracycline, was inactive against $M$. ulcerans in one study [58].

\subsection{Oxazolidinones}

Linezolid showed only intermediate activity with an MIC ranging from 3 to $10 \mu \mathrm{M}$; as posizolid and sutezolid. This oxazolidinone is currently considered as a major component of second-line tuberculosis treatment [58, 65]. In another study with 29 isolates of $M$. ulcerans tested, the mean MIC was $0.73 \mu \mathrm{g} / \mathrm{ml}$ [49].

\subsection{Avermectins}

Avermectins are a class of anti-helminth and anti-parasite drugs that are thought to have intermediate efficacy on $M$. tuberculosis [66]. Subsequent testing showed the MIC for ivermectin and moxidectin ranging from 4 to $8 \mu \mathrm{g} / \mathrm{ml}$ [67]. In another study, the MIC for selamectin was $2-4 \mu \mathrm{g} / \mathrm{ml}$ and ivermectin and moxidectin showed no activity $>32 \mu \mathrm{g} / \mathrm{ml}[68]$.

\subsection{Trimethoprim and Epiroprim}

Trimethoprim was not effective against $M$. ulcerans. However, epiroprim, another dihydrofolate reductase inhibitor, showed an MIC of $0.5-1.0 \mu \mathrm{g} / \mathrm{ml}$ [69].

\section{Experimental Drugs}

A series of experimental compounds, mainly originating from TB drug research pipelines were tested for use in $M$. ulcerans chemotherapy.

Diarylthiazoles and 1,3-diaryltriazenes are compounds with high potency against mycobacteria, including M. ulcerans. Different experimental 1,3-diaryltriazene analogues showed sub-micro molar inhibitory activity against $M$. ulcerans 1615 lux [70]. Similarly, diarylthiazoles, like fatostatin, were found to have a significant efficacy against $M$. ulcerans [58]. KRM-1648, a benzoxazinorifamycin with good efficacy in the TB mouse model was equally tested for use in M. ulcerans and showed 
good killing at an MIC of 12-25 $\mu \mathrm{g} / 1$ [39]. The diarylquinoline bedaquiline, also a TB research drug had a mean MIC of $0.03(0.015-0.12) \mu \mathrm{g} / \mathrm{ml}$ when tested against a set of 29 isolates [49].

GyrB is a common drug target in TB therapy and experimental GyrB inhibitors showed good to moderate activities (MIC 0.3-10 $\mu \mathrm{M}$ ) against $M$. ulcerans [58]. In the same study, pyrrolamide and aminopyrazinamide exhibited activity with an MIC of 0.3-1.0 $\mu \mathrm{M}$ [58]. These investigators found one aminopyrazole, as well as pyrazolopyrimidine and one hydroxyquinolone to kill $M$. ulcerans with MICs from 0.3 to $3.0 \mu \mathrm{M}[58]$.

There is high genetic similarity between M. tuberculosis and M. ulcerans. Yet some of the compounds that are active against the first, failed to prove efficacy in the latter. SQ641, PA-824 a promising nitroimidazopyran for tuberculosis, semisynthetic ketolides (HMR 3647 and HMR 3004), bisbenzaldehydes, as well as quinolinyl pyrimidines and phenothiazines showed only moderate or no antimicrobial effect against $M$. ulcerans (Table 1) [21, 39, 49, 51, 52, 57, 58, 67, 70].

Table 1 Summary of compounds tested against M. ulcerans in vitro

\begin{tabular}{l|l|l}
\hline Compound & Class & Mic in-vitro \\
\hline Rifampin & Rifamycin & $0.1-0.81 \mu \mathrm{g} / \mathrm{ml}$ \\
\hline Rifabutin & Rifamycin & $0.1-0.4 \mu \mathrm{g} / \mathrm{ml}$ \\
\hline Rifapentine & Rifamycin & $0.125-0.5 \mu \mathrm{g} / \mathrm{ml}$ \\
\hline Dapsone & Sulfone & $0.94-4.0 \mathrm{mg} / \mathrm{l}$ \\
\hline Doxycycline & Tetracycline & Inactive \\
\hline Streptomycin & Aminoglycoside & $0.33-1.10 \mu \mathrm{g} / \mathrm{ml}$ \\
\hline Amikacin & Aminoglycoside & $0.5-0.65 \mu \mathrm{g} / \mathrm{ml}$ \\
\hline Azithromycin & Macrolide & $0.39 \mu \mathrm{g} / \mathrm{ml}$ \\
\hline Clarithromycin & Macrolide & $0.125-1.25 \mu \mathrm{g} / \mathrm{ml}$ \\
\hline Ofloxacin & Quinolone & $1.26-2.0 \mu \mathrm{g} / \mathrm{ml}$ \\
\hline Ciprofloxacin & Quinolone & $1.15 \mu \mathrm{g} / \mathrm{ml} ; 1-3 \mathrm{uM}$ \\
\hline Sparfloxacin & Quinolone & $0.25 \mathrm{mg} / 1$ \\
\hline Moxifloxacin & Quinolone & $0.14 \mu \mathrm{g} / \mathrm{ml}$ \\
\hline Sitafloxacin & Quinolone & $0.125-0.5 \mu \mathrm{g} / \mathrm{ml}$ \\
\hline Prulifloxacin & Quinolone & $1-3 \mu \mathrm{M}$ \\
\hline GyrA_NTBI-analog & Topoisomerase II inhibitor & Inactive \\
\hline GYRA-NTBI PubChem_15983305 & Topoisomerase II inhibitor & Inactive \\
\hline GyrB_Pyrollamide & Pyrrolamide & $0.3-1.0 \mu \mathrm{M}$ \\
\hline PubChem_25223515 & & \\
\hline GyrB_Aminopyrazinamide & Aminopyrazinamide & $0.3-1.0 \mu \mathrm{M}$ \\
\hline Oxazolidinone PubChem_10251911 & Oxazolidinone & $0.3-1.0 \mu \mathrm{M}$ \\
\hline Linezolid & Oxazolidinone & $3-10 \mu \mathrm{M} ; 0.73 \mu \mathrm{g} / \mathrm{ml}$ \\
\hline Posizolid & Oxazolidinone & $3-10 \mu \mathrm{M}$ \\
\hline PubChem_10251911 & Oxazolidinone & $0.3-1.0 \mu \mathrm{M}$ \\
\hline Clofazimine & Riminophenazine & $2.19 \mu \mathrm{g} / \mathrm{ml}$ \\
\hline Selamectin & Avermectin & $2-4 \mu \mathrm{g} / \mathrm{ml}$ \\
\hline Ivermectin & Avermectin & $4-32 \mu \mathrm{g} / \mathrm{ml}$ \\
\hline Moxidectin & Avermectin & $4-32 \mu \mathrm{g} / \mathrm{ml}$ \\
\hline & & \\
\hline & & \\
\hline & &
\end{tabular}




\begin{tabular}{l|l|l}
\hline Compound & Class & Mic in-vitro \\
\hline HMR 3647 & Ketolide & $5-40 \mu \mathrm{g} / \mathrm{ml}$ \\
\hline HMR 3004 & Ketolide & $5-40 \mu \mathrm{g} / \mathrm{ml}$ \\
\hline 1,3-diaryltriazenes & & \\
\hline Diarylthiazoles & & \\
\hline SQ641 & & $8 \mu \mathrm{g} / \mathrm{ml}$ \\
\hline KRM-1648 & Benzoxazinorifamycin & $0.012 \mathrm{and} 0.025 \mu \mathrm{g} / \mathrm{ml}$ \\
\hline R207910 & Diarylquinoline & $0.03 \mu \mathrm{g} / \mathrm{ml}$ \\
\hline PA-824 & Nitroimidazopyran & $13.1 \mu \mathrm{g} / \mathrm{ml}$ \\
\hline Aminopyrazoles & & $0.3-1.0 \mu \mathrm{M}$ \\
\hline Pyrazolopyrimidines & & $0.3-1.0 \mu \mathrm{M}$ \\
\hline hydroxyquinolones & & $1-3 \mu \mathrm{M}$ \\
\hline Quinolinyl pyrimidines & & Inactive \\
\hline Phenothiazines & & Inactive \\
\hline Diazene-1,2-dicarboxamides & & $5.67-7.25 \mu \mathrm{g} / \mathrm{ml}$ \\
\hline
\end{tabular}

\section{$4 \quad$ Clinical Studies}

As mentioned above, the shift to antimicrobial therapy was made following the landmark study by Etuaful et al. using $15 \mathrm{mg} / \mathrm{kg}$ body weight intramuscular streptomycin and $10 \mathrm{mg} / \mathrm{kg}$ body weight oral rifampin [29]. The earlier mentioned randomized placebo-controlled study from Côte d'Ivoire with 41 study participants testing a combination of rifampicin and dapsone lacked power and had too many dropouts with only 2 months follow-up [9].

During the past decade, case series and observational cohort studies of patients with BU were reported that we discuss here; case reports will not be discussed as BU patients may occasionally heal spontaneously [71, 72]. One observational cohort study in Benin reported on 224 patients, the majority with large (category III; $>15 \mathrm{~cm}$ ) lesions. $215(96 \%)$ were categorized as treatment successes, and 9, including 1 death and 8 losses to follow-up, were treatment failures. Of the 215 successfully treated patients, $102(47 \%)$ were treated exclusively with antibiotics and 113 (53\%) were treated with antibiotics plus surgical excision and skin grafting; $73 \%$ of patients with lesions of $>15 \mathrm{~cm}$ in diameter underwent surgery, whereas only $17 \%$ of patients with lesions of $<5 \mathrm{~cm}$ had surgery [73]. Compliance with therapy was excellent; 208 of the 215 patients were actively retrieved 1 year after treatment and 3 (1.44\%) of the 208 retrieved patients had recurrence of M. ulcerans disease, 2 among the 107 patients treated only with antibiotics and 1 among the 108 patients treated with antibiotics plus surgery. In a report from Australia, 40 patients received combined antimicrobial and surgical treatment. Failures occurred often but twice as often in the group that received surgery as the only treatment modality [74]. The Australian guidelines of 2006 (published in 2007) still proposed radical surgery including the removal of a rim of apparently healthy tissue for treatment [75]. Several other groups have reported on outcome of combined surgical and antimicrobial treatment-of $79(61 \%)$ patients retrieved, 7 (9\%) had a recurrence [76]. In a series of 92 patients treated in the Bas-Congo, DR Congo, patients received surgery 
and antimicrobial (streptomycin/rifampicin) treatment sometimes more than 8 weeks, with a high success rate (98.4\% in PCR-confirmed patients) and low recurrence rate $(1.1 \%)$.

With a substantial proportion of study participants receiving both surgery and antimicrobial therapy it has been difficult to tease out the potential of antimicrobial treatment alone. In larger lesions, the general assumption is that surgery as added therapy is essential to obtain wound closure; in smaller lesions, the hypothesis that antimicrobial treatment alone without extensive surgical debridement could heal BU with no recurrence, was tested in a controlled clinical trial including 151 study participants with small ( $<10 \mathrm{~cm}$ cross-sectional diameter) lesions, almost all being confirmed by PCR to have $M$. ulcerans infection. After 4 weeks of streptomycin/rifampicin combination therapy, patients were randomized to either continue for 4 more weeks or switch to oral treatment with rifampicin/clarithromycin combination therapy. A switch from streptomycin to clarithromycin after 4 weeks was non-inferior to 8 weeks streptomycin and rifampicin in these early lesions; recurrence or failure was very lowsuccess rates were $96 \%$ in the group receiving 8 weeks of streptomycin/rifampicin, and $91 \%$ in the group that switched to oral treatment which was statistically noninferior [36]. In an observational cohort of 160 PCR-confirmed patients with BU, treated with streptomycin/rifampicin for 8 weeks, 152 healed without surgery; of 158 patients seen 1 year later, no recurrences were noted [77]. In three centers in Ghana, 43 patients - 16 of them having category II/III lesions-received the streptomycin/ rifampicin combination regimen that was switched after 2 weeks to oral rifampicin/ clarithromycin. Ninety-three percent had successful outcome, only one had surgery, and none had recurrence at follow-up [37]. A recent report reflects perhaps better what happens under routine service conditions [78]. Patients $(n=50)$ with confirmed BU in two centers in the Brong-Ahafo Region of Ghana were followed over time; a majority had first used traditional treatment; the patient population consisted predominantly of peasant farmers with no formal education, or children. Only 40 completed treatment and of those, only 28 healed; in the others the lesions reduced in size [78].

In summary, based on the above evidence from various studies and reports-notably, the potential to achieve cure without relapse, at much higher rates than previously with surgery alone, the role of antimicrobial treatment in the management of BU has become standard of care [79]. All drugs used for BU have significant side effects, especially in the elderly [80]; but aminoglycoside drugs—-streptomycin and amikacin—are notoriously the most toxic compounds [81], and although most of the evidence supporting antimicrobial treatment for BU has been provided for the combination of rifampicin and streptomycin, there has been a search for an alternative, fully oral treatment for over a decade now. The first report came from Australia where the patient population is typically elderly and more vulnerable for the ototoxicity of aminoglycosides; the results of a small $(n=4)$ case series [82] was soon followed by larger series of 43 patients that had oral antimicrobials combined with surgery with only one patient that had a relapse [83]. Later, a series of 132 patients had oral therapy with less surgery than before and with excellent healing rates and only one relapse [84]. In Benin, alloral treatment was given for 8 weeks to 30 patients with BU with a slightly higher daily dose of clarithromycin (12 mg/kg once daily, combined with rifampicin $10 \mathrm{mg} / \mathrm{kg}$ ); 
they all healed without relapse [35]. The WHO initiated a randomized clinical trial in 2012, that was recently completed (clinicaltrials.gov identifier: NCT01659437) comparing all-oral, 8-week rifampicin plus clarithromycin (in sustained release, once daily, $15 \mathrm{mg} / \mathrm{kg}$ ) with standard streptomycin/rifampicin treatment; interim analysis showed non-inferiority of the all-oral group compared to the streptomycin/rifampicin group, and in the bi-annual meeting on BU in Geneva in the Spring of 2017, it was decided that sufficient evidence had been provided to change the treatment recommendation to all-oral treatment, using rifampicin and clarithromycin. ${ }^{2}$ The Australian guidelines had already recommended all-oral antimicrobial treatment [85] earlier.

\subsection{Secondary Infection}

Wounds caused by $M$. ulcerans often harbor a multitude of secondary pathogens. Isolates of S. aureus, Aeromonas hydrophila, Pseudomonas aeruginosa and Klebsiella pneumoniae with a high degree of resistance to commonly used antibiotics were found in BU lesions in Ghana and Nigeria [86, 87]. S. aureus isolated from M. ulcerans lesions were found to harbor a large array of virulence factors. In Ghana, an association between the presence of these organisms and delayed healing was observed. The question whether these secondary bacterial invading pathogens are merely bystanders of $M$. ulcerans infection or actual contributors has not been resolved [88]. Apart from the question whether these secondary invaders inhibit and delay wound healing or not, rational use of antimicrobials other than the combination recommended by WHO, should be discouraged, in order to prevent further antimicrobial selection pressure and the further emergence of resistance among these secondary organisms [89]. The skin microbiome was significantly changed, however it is not clear if and how this contributes to pathology [90]. The option to explore topical instead of systemic antimicrobial agents, e.g., nitric oxide needs perhaps further attention [91]. For more detailed information the reader is referred to chapter "Secondary Infection of Buruli Ulcer Lesions" of this book.

\subsection{HIV Co-infection}

HIV co-infection complicates diagnosis and treatment of BU [92]. In 2015, a consensus statement was published by WHO; HIV testing is recommended for all patients with BU. For more detailed information the reader is referred to chapter "Management of BU-HIV Co-infection" of this book. Combined antiretroviral treatment might be postponed 4 weeks after starting streptomycin/rifampicin treatment for BU, because of drug-drug interactions and adverse drug effects, and the importance to use the most effective bactericidal drug combination available for $M$. ulcerans infection. ${ }^{3}$

\footnotetext{
${ }^{2}$ http://www.who.int/neglected_diseases/events/WHO_BU_TAG_2017_report.pdf?ua $=1$. ${ }^{3}$ http://apps.who.int/iris/bitstream/10665/154241/1/WHO_HTM_NTD_IDM_2015.01_eng. pdf?ua $=1$.
} 


\section{Conclusions; Areas of Uncertainty; and Future Directions}

There is no doubt that antimicrobial therapy-preferably, all-oral rifampicin-based treatment is essential for cure without relapse. Not all questions have been resolved, however. Despite the overwhelming evidence that the combination of clarithromycin and rifampicin works well, with acceptable levels of toxicity especially in the relatively young patient population in West Africa, the dosage of these drugs has been different across different studies and regions. Drug-drug interactions of rifampicin and clarithromycin are complex-rifampicin induces the metabolism of clarithromycin into its inactive metabolite 14-OH clarithromycin; and it induces its own hepatic elimination as well. Clarithromycin inhibits the elimination of rifampicin [93]. Increased drug exposure to rifampicin is much safer than previously thought [94].

An important next step could be to improve the dosage by extensive pharmacokinetic modeling; a fixed dose combination drug might have considerable advantage for compliance, logistics and in order to prevent monotherapy; this approach has been shown to be highly successful in tuberculosis, malaria and HIV infection.

Further, duration of therapy has not been individualized; based on the initial observations in the small study by Etuaful et al. [29], where no viable M. ulcerans bacilli were recovered from lesions in patients treated for at least 4 weeks, an arbitrary safety margin of 4 more weeks was chosen. The question whether larger lesions need longer treatment, or smaller lesions perhaps less has not been addressed in well-designed studies. In a follow-up study among 56 patients in Ghana, who defaulted before the end of planned treatment, $92 \%$ of patients with category I lesions $(<5 \mathrm{~cm})$ were healed with treatment duration of 32 days or more [95], suggesting that smaller lesions indeed might do well with much less than standard 8 -weeks treatment.

Next, the treatment of BU extends well beyond administration of antimicrobials alone. During the active phase of the disease adequate wound care is imperative to ensure healing and to prevent disabling scarring. Non-adhesive, absorbent dressing materials have been shown to improve the wound microenvironment and improve time to healing [96, 97], and might also play a role to prevent painful dressing changes. While M. ulcerans is generally thought to be a pain-free disease as the toxin mycolactone causes hypoalgesia [98-100], later on, as lesions start to heal and the mycolactone is washed out from the lesions and the system, patients report considerable pain especially during wound dressing changes and during physiotherapy needed to prevent and treat contractures and disability [101]. For patient comfort and compliance, a sound wound-care program is essential, with adequate pain management [102].

During and after the completion of antimicrobial treatment, many patients still experience restrictions through either physical disability caused by wound contractures or joint involvement of lesions; or psycho-social participation restrictions caused by social stigmatization [103, 104]. Physical therapy and societal inclusion and de-stigmatization are therefore important components of BU control programs 
[81, 105]. Community-based approaches with early case finding are essential to prevent the large disease burden in individuals and communities [106, 107].

Future research thus should focus on evaluating shorter treatment for limited lesions while supplementing standard treatment with clofazimine or fluoroquinolones to provide a treatment tailored to the lesion size and estimated bacterial burden of individual patients.

Such attempts have so far been hampered by the fact that no good measurable surrogate parameter of treatment success, such as a blood marker, is available. However recent advances in the use of mass-spectrometry to carefully measure mycolactone in patient samples could aid in this regard.

Furthermore, there is dire need for the integration of control programs for tropical skin conditions such as BU, yaws, leprosy, etc. on a public health level [108]. Health officials should identify opportunities for systematic integration for the control of tropical neglected diseases depending on the prevalent diseases and available resources in any given setting.

Finally, BU treatment should be accompanied by surgery where needed, as well as good wound care, analgesia, physiotherapy and advocacy to reduce stigma.

\section{References}

1. MacCallum P, Tolhurst JC (1948) A new mycobacterial infection in man. J Pathol Bacteriol 60(1):93-122

2. Daniel TM (2006) The history of tuberculosis. Respir Med 100(11):1862-1870

3. Daniels M, Hill AB (1952) Chemotherapy of pulmonary tuberculosis in young adults; an analysis of the combined results of three Medical Research Council trials. Br Med J 1(4769):1162-1168

4. Schatz A, Waksman SA (2016) Effect of streptomycin and other antibiotic substances upon mycobacterium tuberculosis and related organisms. Proc Soc Exp Biol Med 57(2):244-248

5. Meyers WM, Shelly WM, Connor DH (1974) Heat treatment of Mycobacterium ulcerans infections without surgical excision. Am J Trop Med Hyg 23(5):924-929

6. Stanford JL, Phillips I (1972) Rifampicin in experimental Mycobacterium ulcerans infection. J Med Microbiol 5(1):39-45

7. Revill WD et al (1973) A controlled trial of the treatment of Mycobacterium ulcerans infection with clofazimine. Lancet 2(7834):873-877

8. Fehr H, Egger M, Senn I (1994) Cotrimoxazol in the treatment of Mycobacterium ulcerans infection (Buruli ulcer) in West Africa. Trop Dr 24(2):61-63

9. Espey DK et al (2002) A pilot study of treatment of Buruli ulcer with rifampin and dapsone. Int J Infect Dis 6(1):60-65

10. Portaels F et al (1998) In vitro susceptibility of Mycobacterium ulcerans to clarithromycin. Antimicrob Agents Chemother 42(8):2070-2073

11. van der Werf TS et al (1999) Mycobacterium ulcerans infection. Lancet 354(9183):1013-1018

12. George KM et al (1999) Mycolactone: a polyketide toxin from Mycobacterium ulcerans required for virulence. Science 283(5403):854-857

13. Hockmeyer WT et al (1978) Further characterization of Mycobacterium ulcerans toxin. Infect Immun 21(1):124-128

14. Read JK et al (1974) Cytotoxic activity of Mycobacterium ulcerans. Infect Immun 9(6):1114-1122 
15. Pimsler Met al (1988) Immunosuppressive properties of the soluble toxin from Mycobacterium ulcerans. J Infect Dis 157(3):577-580

16. Debacker $M$ et al (2005) Buruli ulcer recurrence, Benin. Emerg Infect Dis 11(4):584-589

17. Kanga JM et al (2003) Recurrence after surgical treatment of Buruli ulcer in Cote d'Ivoire. Bull Soc Pathol Exot 96(5):406-409

18. Teelken MA et al (2003) Buruli ulcer: differences in treatment outcome between two centres in Ghana. Acta Trop 88(1):51-56

19. Phanzu DM et al (2006) Mycobacterium ulcerans disease (Buruli ulcer) in a rural hospital in Bas-Congo, Democratic Republic of Congo, 2002-2004. Am J Trop Med Hyg 75(2):311-314

20. Amofah G, Asamoah S, Afram-Gyening C (1998) Effectiveness of excision of pre-ulcerative Buruli lesions in field situations in a rural district in Ghana. Trop Dr 28(2):81-83

21. Thangaraj HS et al (2000) In vitro activity of ciprofloxacin, sparfloxacin, ofloxacin, amikacin and rifampicin against Ghanaian isolates of Mycobacterium ulcerans. J Antimicrob Chemother 45(2):231-233

22. Guarner J et al (2003) Histopathologic features of Mycobacterium ulcerans infection. Emerg Infect Dis 9(6):651-656

23. Nienhuis WA et al (2012) Paradoxical responses after start of antimicrobial treatment in Mycobacterium ulcerans infection. Clin Infect Dis 54(4):519-526

24. O'Brien DP et al (2009) Paradoxical immune-mediated reactions to Mycobacterium ulcerans during antibiotic treatment: a result of treatment success, not failure. Med J Aust 191(10):564-566

25. Ruf MT et al (2011) Secondary Buruli ulcer skin lesions emerging several months after completion of chemotherapy: paradoxical reaction or evidence for immune protection? PLoS Negl Trop Dis 5(8):e1252

26. Dega $\mathrm{H}$ et al (2000) Activities of several antimicrobials against Mycobacterium ulcerans infection in mice. Antimicrob Agents Chemother 44(9):2367-2372

27. Dega $\mathrm{H}$ et al (2002) Bactericidal activity of rifampin-amikacin against Mycobacterium ulcerans in mice. Antimicrob Agents Chemother 46(10):3193-3196

28. Marsollier L, Prévot G et al (2003) Susceptibility of Mycobacterium ulcerans to a combination of amikacin/rifampicin. Int J Antimicrob Agents 22(6):562-566

29. Etuaful $\mathrm{S}$ et al (2005) Efficacy of the combination rifampin-streptomycin in preventing growth of Mycobacterium ulcerans in early lesions of Buruli ulcer in humans. Antimicrob Agents Chemother 49(8):3182-3186

30. Johnson PDR et al (2005) Buruli ulcer (M. ulcerans infection): new insights, new hope for disease control. PLoS Med 2(4):e108

31. Radford AJ (2009) The surgical management of lesions of ulcerans infections due to Mycobacterium ulcerans, revisited. Trans R Soc Trop Med Hyg 103(10):981-984

32. van der Werf TS et al (2005) Mycobacterium ulcerans disease. Bull World Health Organ 83(10):785-791

33. Wansbrough-Jones M, Phillips R (2006) Buruli ulcer: emerging from obscurity. Lancet 367(9525): $1849-1858$

34. Doig KD et al (2012) On the origin of Mycobacterium ulcerans, the causative agent of Buruli ulcer. BMC Genomics 13(1):258

35. Chauty A et al (2011) Oral treatment for Mycobacterium ulcerans infection: results from a pilot study in Benin. Clin Infect Dis 52(1):94-96

36. Nienhuis WA et al (2010) Antimicrobial treatment for early, limited Mycobacterium ulcerans infection: a randomised controlled trial. Lancet 375(9715):664-672

37. Phillips RO et al (2014) Clinical and bacteriological efficacy of rifampin-streptomycin combination for two weeks followed by rifampin and clarithromycin for six weeks for treatment of Mycobacterium ulcerans disease. Antimicrob Agents Chemother 58(2):1161-1166

38. Almeida D et al (2011) Activities of rifampin, Rifapentine and clarithromycin alone and in combination against Mycobacterium ulcerans disease in mice. PLoS Negl Trop Dis 5(1): e933 
39. Dhople AM (2001) In vitro activity of KRM-1648, either singly or in combination with ofloxacin, against Mycobacterium ulcerans. Int J Antimicrob Agents 17(1):57-61

40. Owusu E et al (2017) In vitro susceptibility of Mycobacterium ulcerans isolates to selected antimicrobials. Can J Inf Dis Med Microbiol 2017(4):5180984-5180986

41. Chauffour A et al (2016) Sterilizing activity of fully oral intermittent regimens against Mycobacterium ulcerans infection in Mice. PLoS Negl Trop Dis 10(10):e0005066

42. Almeida DV et al (2013) Bactericidal activity does not predict sterilizing activity: the case of rifapentine in the murine model of Mycobacterium ulcerans disease. PLoS Negl Trop Dis 7(2): 2085

43. Beissner $\mathrm{M}$ et al (2010) A genotypic approach for detection, identification, and characterization of drug resistance in Mycobacterium ulcerans in clinical samples and isolates from Ghana. Am J Trop Med Hyg 83(5):1059-1065

44. Jansson $\mathrm{M}$ et al (2014) Comparison of two assays for molecular determination of rifampin resistance in clinical samples from patients with Buruli ulcer disease. J Clin Microbiol 52(4):1246-1249

45. Marsollier L, Honoré N et al (2003) Isolation of three Mycobacterium ulcerans strains resistant to rifampin after experimental chemotherapy of mice. Antimicrob Agents Chemother 47(4):1228-1232

46. Feldman WH, Karlson AG (1957) Mycobacterium ulcerans infections; response to chemotherapy in mice. Am Rev Tuberc 75(2):266-279

47. Pattyn SR, Royackers J (1965) Treatment of experimental infection with mycobacterium leprae in mice. Ann Soc Belg Med Trop 45:27-30

48. Lefrançois $\mathrm{S}$ et al (2007) Curing Mycobacterium ulcerans infection in mice with a combination of rifampin-streptomycin or rifampin-amikacin. Antimicrob Agents Chemother 51(2):645-650

49. Ji B et al (2006) In vitro and in vivo activities of rifampin, streptomycin, amikacin, moxifloxacin, R207910, linezolid, and PA-824 against Mycobacterium ulcerans. Antimicrob Agents Chemother 50(6): 1921-1926

50. Klis S, Stienstra Y, Phillips RO, Abass KM, Tuah W, van der Werf TS (2014) Long term streptomycin toxicity in the treatment of Buruli ulcer: follow-up of participants in the BURULICO drug trial. PLoS Negl Trop Dis 8(3):e2739

51. Dubuisson $T$ et al (2010) In vitro antimicrobial activities of capuramycin analogues against non-tuberculous mycobacteria. J Antimicrob Chemother 65(12):2590-2597

52. Rastogi $\mathrm{N}$ et al (2000) In vitro activities of the ketolides telithromycin (HMR 3647) and HMR 3004 compared to those of clarithromycin against slowly growing mycobacteria at $\mathrm{pHs}$ 6.8 and 7.4. Antimicrob Agents Chemother 44(10):2848-2852

53. Bentoucha A et al (2001) Activities of new macrolides and fluoroquinolones against Mycobacterium ulcerans infection in mice. Antimicrob Agents Chemother 45(11):3109-3112

54. O'Brien DP et al (2012) Successful outcomes with oral fluoroquinolones combined with rifampicin in the treatment of Mycobacterium ulcerans: an observational cohort study. PLoS Negl Trop Dis 6(1):e1473

55. Principi N, Esposito S (2015) Appropriate use of fluoroquinolones in children. Int J Antimicrob Agents 45(4):341-346

56. Treggiari MM et al (2011) Comparative efficacy and safety of 4 randomized regimens to treat early Pseudomonas aeruginosa infection in children with cystic fibrosis. Arch Pediatr Adolesc Med 165(9):847-856

57. Dhople AM, Namba K (2002) In vitro activity of sitafloxacin (DU-6859a) alone, or in combination with rifampicin, against Mycobacterium ulcerans. J Antimicrob Chemother 50(5):727-729

58. Scherr N, Pluschke G, Panda M (2016) Comparative study of activities of a diverse set of antimycobacterial agents against mycobacterium tuberculosis and Mycobacterium ulcerans. Antimicrob Agents Chemother 60(5):3132-3137

59. Namboodiri SS et al (2011) Quinolone resistance in Escherichia coli from Accra, Ghana. BMC Microbiol 11(1):44 
60. Grosset JH et al (2013) Assessment of clofazimine activity in a second-line regimen for tuberculosis in mice. Am J Respir Crit Care Med 188(5):608-612

61. Lechartier B, Cole ST (2015) Mode of action of clofazimine and combination therapy with benzothiazinones against Mycobacterium tuberculosis. Antimicrob Agents Chemother 59(8):4457-4463

62. Arbiser JL, Moschella SL (1995) Clofazimine: a review of its medical uses and mechanisms of action. J Am Acad Dermatol 32(2 Pt 1):241-247

63. Converse PJ et al (2015) Efficacy of rifampin plus clofazimine in a Murine model of Mycobacterium ulcerans disease. PLoS Negl Trop Dis 9(6):e0003823

64. Hwang TJ et al (2014) Safety and availability of clofazimine in the treatment of multidrug and extensively drug-resistant tuberculosis: analysis of published guidance and meta-analysis of cohort studies. BMJ Open 4(1):e004143

65. Bolhuis MS et al (2015) Linezolid tolerability in multidrug-resistant tuberculosis: a retrospective study. Eur Respir J 46(4):1205-1207

66. Lim LE et al (2013) Anthelmintic avermectins kill Mycobacterium tuberculosis, including multidrug-resistant clinical strains. Antimicrob Agents Chemother 57(2):1040-1046

67. Omansen TF et al (2015) In-vitro activity of avermectins against Mycobacterium ulcerans. PLoS Negl Trop Dis 9(3):e0003549

68. Scherr N et al (2015) Selamectin is the avermectin with the best potential for Buruli ulcer treatment. PLoS Negl Trop Dis 9(8):e0003996

69. Dhople AM (2001) Antimicrobial activities of dihydrofolate reductase inhibitors, used singly or in combination with dapsone, against Mycobacterium ulcerans. J Antimicrob Chemother 47(1):93-96

70. Cappoen D et al (2014) Biological evaluation of diazene derivatives as anti-tubercular compounds. Eur J Med Chem 74:85-94

71. Beissner M et al (2012) Spontaneous clearance of a secondary Buruli ulcer lesion emerging ten months after completion of chemotherapy--a case report from Togo. PLoS Negl Trop Dis 6(7): 1747

72. Gordon CL et al (2011) Spontaneous clearance of Mycobacterium ulcerans in a case of Buruli ulcer. PLoS Negl Trop Dis 5(10):e1290

73. Chauty A et al (2007) Promising clinical efficacy of streptomycin-rifampin combination for treatment of buruli ulcer (Mycobacterium ulcerans disease). Antimicrob Agents Chemother 51(11):4029-4035

74. O'Brien DP et al (2007) Outcomes for Mycobacterium ulcerans infection with combined surgery and antibiotic therapy: findings from a South-Eastern Australian case series. Med J Aust 186(2):58-61

75. Johnson PDR et al (2007) Consensus recommendations for the diagnosis, treatment and control of Mycobacterium ulcerans infection (Bairnsdale or Buruli ulcer) in Victoria, Australia. Med J Aust 186:64-68

76. Schunk M et al (2009) Outcome of patients with buruli ulcer after surgical treatment with or without antimycobacterial treatment in Ghana. Am J Trop Med Hyg 81(1):75-81

77. Sarfo FS et al (2010) Clinical efficacy of combination of rifampin and streptomycin for treatment of Mycobacterium ulcerans disease. Antimicrob Agents Chemother 54(9):3678-3685

78. Iddrisah FN et al (2016) Outcome of Streptomycin-Rifampicin treatment of Buruli Ulcer in two Ghanaian districts. Pan Afr Med J 25(Suppl 1):13

79. Johnson PDR (2010) Should antibiotics be given for Buruli ulcer? Lancet 375(9715): 618-619

80. O'Brien DP et al (2017) Antibiotic complications during the treatment of Mycobacterium ulcerans disease in Australian patients. Intern Med J 47(9):1011-1019

81. Klis S, Ranchor A et al (2014) Good quality of life in former Buruli ulcer patients with small lesions: long-term follow-up of the BURULICO trial. PLoS Negl Trop Dis 8(7):e2964 
82. Gordon CL et al (2010) All-oral antibiotic treatment for Buruli ulcer: a report of four patients. PLoS Negl Trop Dis 4(11):e770

83. Friedman ND et al (2013) Mycobacterium ulcerans disease: experience with primary oral medical therapy in an Australian cohort. PLoS Negl Trop Dis 7(7):e2315

84. Friedman ND et al (2016) Increasing experience with primary oral medical therapy for Mycobacterium ulcerans disease in an Australian cohort. Antimicrob Agents Chemother 60(5):2692-2695

85. O'Brien DP, Jenkin G et al (2014) Treatment and prevention of Mycobacterium ulcerans infection (Buruli ulcer) in Australia: guideline update. Med J Aust 200(5):267-270

86. Amissah NA et al (2015) Genetic diversity of Staphylococcus aureus in Buruli ulcer. PLoS Negl Trop Dis 9(2):e0003421

87. Yeboah-Manu D et al (2013) Secondary bacterial infections of buruli ulcer lesions before and after chemotherapy with streptomycin and rifampicin. PLoS Negl Trop Dis 7(5):e2191

88. Amissah NA et al (2017) Virulence potential of Staphylococcus aureus isolates from Buruli ulcer patients. Int J Med Microbiol 307(4-5):223-232

89. Barogui YT et al (2013) Towards rational use of antibiotics for suspected secondary infections in Buruli ulcer patients. PLoS Negl Trop Dis 7(1):e2010

90. Van Leuvenhaege $\mathrm{C}$ et al (2017) Bacterial diversity in Buruli ulcer skin lesions: Challenges in the clinical microbiome analysis of a skin disease. PLoS ONE 12(7):e0181994

91. Phillips R et al (2004) Pilot randomized double-blind trial of treatment of Mycobacterium ulcerans disease (Buruli ulcer) with topical nitrogen oxides. Antimicrob Agents Chemother 48(8):2866-2870

92. O’Brien DP, Ford N et al (2014) Management of BU-HIV co-infection. Tropical Med Int Health 19(9):1040-1047

93. Alffenaar JWC et al (2010) Pharmacokinetics of rifampin and clarithromycin in patients treated for Mycobacterium ulcerans infection. Antimicrob Agents Chemother 54(9):3878-3883

94. Boeree $\mathrm{MJ}$ et al (2015) A dose-ranging trial to optimize the dose of rifampin in the treatment of tuberculosis. Am J Respir Crit Care Med 191(9):1058-1065

95. Klis S et al (2016) Clinical outcomes of Ghanaian Buruli ulcer patients who defaulted from antimicrobial therapy. Tropical Med Int Health 21(9):1191-1196

96. Velding $\mathrm{K}$ et al (2016) The application of modern dressings to Buruli ulcers: results from a pilot implementation project in Ghana. Am J Trop Med Hyg 95(1):60-62

97. Velding K et al (2014) Wound care in Buruli ulcer disease in Ghana and Benin. Am J Trop Med Hyg 91(2):313-318

98. Anand U et al (2016) Mycolactone-mediated neurite degeneration and functional effects in cultured human and rat DRG neurons: mechanisms underlying hypoalgesia in Buruli ulcer. Mol Pain 12:1744806916654144

99. En J et al (2017) Mycolactone cytotoxicity in Schwann cells could explain nerve damage in Buruli ulcer. PLoS Negl Trop Dis 11(8):e0005834

100. En J et al (2008) Mycolactone is responsible for the painlessness of Mycobacterium ulcerans infection (Buruli ulcer) in a murine study. Infect Immun 76(5):2002-2007

101. de Zeeuw J et al (2015) Assessment and treatment of pain during treatment of Buruli ulcer. PLoS Negl Trop Dis 9(9):e0004076

102. Alferink M et al (2015) Pain associated with wound care treatment among Buruli ulcer patients from Ghana and Benin. S. C. Hausmann-Muela, ed. PLoS ONE 10(6):e0119926

103. Renzaho AMN et al (2007) Community-based study on knowledge, attitude and practice on the mode of transmission, prevention and treatment of the Buruli ulcer in Ga West District, Ghana. Tropical Med Int Health 12(3):445-458

104. Stienstra $Y$ et al (2005) Factors associated with functional limitations and subsequent employment or schooling in Buruli ulcer patients. Tropical Med Int Health 10(12): $1251-1257$ 
105. de Zeeuw J et al (2014) Psychometric properties of the participation scale among former Buruli ulcer patients in Ghana and Benin. PLoS Negl Trop Dis 8(11):e3254

106. Abass KM et al (2015) Buruli ulcer control in a highly endemic district in Ghana: role of community-based surveillance volunteers. Am J Trop Med Hyg 92(1):115-117

107. Barogui YT et al (2014) Contribution of the community health volunteers in the control of Buruli ulcer in Bénin. PLoS Negl Trop Dis 8(10):e3200

108. Mitjà $\mathrm{O}$ et al (2017) Integrated control and management of neglected tropical skin diseases. PLoS Negl Trop Dis 11(1):e005136

Open Access This chapter is licensed under the terms of the Creative Commons Attribution 4.0 International License (http://creativecommons.org/licenses/by/4.0/), which permits use, sharing, adaptation, distribution and reproduction in any medium or format, as long as you give appropriate credit to the original author(s) and the source, provide a link to the Creative Commons license and indicate if changes were made.

The images or other third party material in this chapter are included in the chapter's Creative Commons license, unless indicated otherwise in a credit line to the material. If material is not included in the chapter's Creative Commons license and your intended use is not permitted by statutory regulation or exceeds the permitted use, you will need to obtain permission directly from the copyright holder. 